\title{
Análise sociométrica da rede de relacionamento das bibliotecas que constituem o Consórcio das Universidades Federais do Sul-Sudeste de Minas Gerais
}

Nivaldo Oliveira

Mestre em Administração pela Universidade Federal de Lavras.Especialista em Gestão do Conhecimento e Tecnologia da Informação e graduado em Biblioteconomia pelo Centro Universitário de Formiga (UNIFOR/MG).Bibliotecário da Universidade Federal de Lavras, MG

Donizeti Leandro de Souza

Doutorando em Administração pela Universidade Federal de Lavras. Especialista em Gestão de pessoas pela Universidade Federal de Itajubá e em Gestão Executiva de Negócios pela PUC Minas Gerais. Graduado em Administração de Empresas com ênfase em gestão de negócios pela Universidade do Vale do Sapucaí

Cleber Carvalho de Castro

Doutorado em Agronegócios pela Universidade Federal do Rio Grande do Sul. Mestrado em Administração pela Universidade Federal do Rio Grande do Sul. Graduado em Administração pela Universidade Federal de Lavras. Professor Adjunto da Universidade Federal de Lavras

O objetivo deste trabalho foi identificar a estrutura da rede de relacionamentos das Bibliotecas que constituem o Consórcio das Universidades Federais do Sul-Sudeste de Minas Gerais e verificar quais instituições se mostram mais centrais na estrutura de relacionamentos. Para isto, foi adotada a metodologia de estudo de caso, considerando abordagens qualitativas (aspectos relacionais) e quantitativas (indicadores estruturais), com

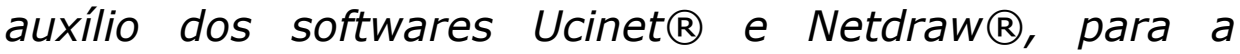
elaboração das matrizes sociométricas, proporcionando análises de centralidade de cada ator na rede, a presença de laços fracos/fortes e a existência de buracos 
estruturais na rede. Como resultado de pesquisa, foi evidenciada grande centralidade da UFJF e UFV, o que representa uma posição favorável para a busca e trocas de informações com melhores condições para gerar inovação na rede.

Palavras-chave: Biblioteca universitária; Análise sociométrica; Centralidade; Análise de redes sociais; Redes interorganizacionais.

\section{Sociometric analysis of the relationship network of libraries that make up the Consortium of Federal Universities of the South-Southeast of Minas Gerais}

The aim of this study was to identify the structure of the social network of libraries that make up the Consortium of Federal Universities of the South-Southeast of Minas Gerais and see which institutions are more central in the structure of relationships. For this, we adopted a case study methodology, considering qualitative approaches (relational aspects) and quantitative (structural indicators) with the aid of software Ucinet Netdraw $($ and ( ) for the preparation of sociometric matrices, providing analysis of centrality of each actor in the network the presence of weak links / strong and the existence of structural holes in the network. As a result of research, it was evident the centrality of great UFJF and UFV, which represents a favorable position for the search and exchange information with better conditions to generate innovation in the network.

Keywords: $\quad$ university library. Sociometric analysis. Centralization. Social network analysis. Interorganizational networks.

Recebido em 29.05.2013 Aceito em 10.06.2013

\section{Introdução}

Devido ao aumento da competição no mercado e a busca incessante da excelência organizacional e resultados, atuar isoladamente tornou-se 
complexo e, muitas vezes, desfavorável. A lógica predominante das redes está vinculada à possibilidade de ganhos para todos os integrantes de um negócio, pois o foco das decisões passou de uma posição individual para coletiva, na busca de inovações, avanços e aceleração da produtividade, que pode ser gerada por meio de estruturas em redes (BRITTO, 2002).

O termo rede em si é uma noção abstrata, que se refere a um conjunto de nós conectados por relacionamentos. Várias empresas, organizações ou mesmo pessoas aglomeram-se de determinada forma para obter resultados e contatos que poderão gerar possibilidades não adquiridas, quando se trabalha isoladamente. Diante do exposto, podemos levantar as seguintes indagações que delineiam esta pesquisa: qual a estrutura da rede de relacionamento das bibliotecas que constituem 0 Consórcio das Universidades Federais do Sul-Sudeste de Minas Gerais? Qual a importância dos atores centrais na rede?

Como forma de encontrar respostas a estas questões, propõe-se, neste estudo, a realização de uma análise sociométrica da estrutura da rede de relacionamento das bibliotecas que constituem o Consórcio das Universidades Federais do Sul-Sudeste de Minas Gerais e verificar a importância dos atores centrais na rede. Assim, partindo de uma análise estrutural, busca-se medir e comparar o nível de interação entre universidades e identificar, por meio de indicadores, as bibliotecas que expressam maior representatividade na rede.

O texto apresenta um referencial teórico sobre redes interorganizacionais, destacando os principais conceitos e a importância de atuar em rede. Nesse tópico, também são apresentadas duas importantes teorias explicativas da análise estrutural: a teoria dos buracos estruturais (BURT, 1992) e a teoria dos laços fortes e fracos (GRANOVETTER, 1973). Posteriormente, apresenta-se uma breve revisão sobre sociometria e análise de redes sociais, seguido de uma contextualização do consórcio das universidades federais do sul-sudeste de Minas Gerais. Em seguida, apresenta-se a metodologia utilizada no estudo e, por fim, os resultados da análise, conclusões e as possíveis limitações/sugestões de novos estudos.

\section{Redes interorganizacionais}

Nohria, já em 1992, detectava um crescimento vertiginoso de estudos envolvendo redes interorganizacionais, o qual, segundo Nohria (1992), deve-se a três fatores principais: (1) amadurecimento do tema na área acadêmica; (2) desenvolvimentos tecnológicos (aumento da produção e de novas tecnologias); e (3) nova competição (construção de relacionamentos horizontais e conexões entre empresas).

Estudos envolvendo redes interorganizacionais têm evidenciado a cooperação como um importante fator de competitividade, transcendendo a perspectiva individual para relações coletivas entre empresas e instituições. Entre os benefícios, tem-se a troca de experiências, acesso a tecnologias, compartilhamento de canais e fornecedores, maior acesso a 
informações e abertura de mercados (BENGTSON; KOCK, 1999; BRITTO, 2002; MA, 2004; LACOSTE, 2012). Neste sentido, Fombrun (1982) considera o termo "rede" uma noção abstrata, que, por si só, refere-se a um conjunto de enredos e relacionamentos que se conectam através de diversas dimensões: sociais, econômicas e organizacionais, sob diferentes propriedades de coordenação.

Já Alejandro e Norman (2006) conceituam redes como um conjunto de nós (atores), vínculos (relações) e fluxos. Sendo assim, uma rede representa um grupo de atores que, de forma agrupada ou individual, se relacionam uns com os outros com um fim específico, caracterizando-se pela existência de fluxos de informação. Esses fluxos podem assumir relações orientadas, quando há a transmissão de bens, serviços, etc., de um ator para outro; ou não orientadas, quando não há existência de transmissões unilaterais entre atores, mas, sim, uma relação que não comporta qualquer tipo de orientação (LIMIEUX; OUIMET, 2008).

Neste sentido, percebe-se que 0 objetivo das redes interorganizacionais é reunir atributos que permitam uma adequação ao ambiente competitivo, por meio de uma estrutura dinâmica que utilize ações uniformizadas, descentralizadas e que evitem que as empresas envolvidas percam sua flexibilidade e mantenha sua competitividade no mercado (BALESTRIN; VERSCHOORE; REYES JUNIOR, 2010; NOHRIA, 1992).

Dentre as variáveis que impactam na formação de redes, Grandori e Soda (1995) destacam: (1) o número de unidades a serem coordenadas, (2) o grau de diferenciação entre organizações, (3) intensidade da interdependência, (4) flexibilidade, (5) grau de negociação e dependência de recursos. Destacam, ainda ,mecanismos de comunicação, decisão e processos de negociação, integração de unidades, equipes de apoio, relações de poder (hierarquia/autoridade), planejamento, sistema de incentivo e controle social.

Nesta perspectiva de estudo, duas teorias têm contribuído muito para os estudos em redes: a teoria da força dos laços fracos (GRANOVETTER, 1973) e a teoria dos buracos estruturais (BURT, 1992). Juntas, essas teorias trazem importantes conceitos para o entendimento de relações e estruturas sociais geradas por organizações ligadas em rede

\subsection{Teoria dos laços fortes e fracos}

No que diz respeito à construção dos laços, Granovetter (1973) salienta que a força e a intensidade destes podem variar entre fortes (estreitos), que se caracterizam pela intimidade e pela proximidade; e fracos (frouxos), que se caracterizam por relações esparsas e não traduzem proximidade e intimidade.

$\mathrm{Na}$ medida em que se tornam mais fortes, os laços de relacionamento e a interação interpessoal entre agentes, mais sólidos, serão a base de confiança, de comprometimento e das responsabilidades na estrutura da rede social. Porém, as redes dos laços fortes tendem a se 
fechar sobre si mesmos, possibilitando menos circulação de novas informações, valorizando os laços fracos, como forma de lançar pontes entre atores capazes de fluir maior comunicação de informações nos diversos canais da rede (LIMIEUX; QUIMET, 2008; CAPALDO, 2007).

Segundo Wu (2012), estabelecer um laço fraco é o primeiro e mais fácil, em qualquer rede de relacionamento. Além de parentesco, quase todas as outras relações sociais iniciam-se a partir de laços fracos. Mesmo as relações de parentescos começam de laços fracos e é somente através dos encontros familiares frequentes e interações que é possível que esses laços se desenvolvam em relacionamentos mais fortes. Considerando que os laços fracos são importantes e valiosos para uma rede, quando se trata da diversidade e não da intensidade, a questão é: como evoluir de laços fracos para laços fortes?

Já o valor dos laços fortes está no relacionamento e seu valor é muito maior do que qualquer quantidade de laços fracos que se possa conquistar, pois os laços fortes incluem a importância da cooperação e da reciprocidade, promovendo assim a confiança mútua e a abertura para a troca de conhecimentos intensivos (WU, 2012; CAPALDO, 2007).

\subsection{Teoria dos buracos estruturais}

Um debate clássico sobre redes como elos tem sido discutido a partir de Burt (1992), com base na teoria dos Buracos Estruturais, pelo fato de serem considerados como uma forma valiosa de capital social. Trabalhos atuais (BURT; KILDUFF; TASSELLI, 2013; CASANUEVA; CASTRO; GALÁN, 2013) indicam que o tamanho da rede de uma organização pode ser considerado um fator importante para a inovação, através do grau de conectividade ou a falta dela, entre os parceiros. Segundo o autor, uma posição na rede pode estar melhor situada, quando determinados atores encontram conectados a outros atores não conectados entre si, gerando mais benefícios e oportunidades.

Buracos estruturais são lacunas nos fluxos de informações entre empresas ligadas ao mesmo ator, mas não vinculados a outros. Um buraco estrutural indica que os atores de cada extremidade do buraco podem ter acesso a diferentes fluxos de informações (HARGADON; SUTTON, 1997). Neste sentido, um "buraco estrutural" pode mudar o fluxo e o sentido da circulação da informação (FAGERBERG; MOWERY; NELSON, 2006).

No que tange à teoria de buraco estrutural, o foco está principalmente nos atores da rede que mantém possibilidade de obter parceiros não ligados entre si, mais do que atores envolvidos em redes densamente conexas (AARSTAD, 2012). Os buracos estruturais podem trazer outros benefícios no que se refere ao volume, à inovação e à qualidade das informações que circulam em rede de atores mais conectados (BALESTRO, 2002). 


\section{Sociometria e análise de redes sociais}

A análise de redes sociais teve início em 1934, nos Estados Unidos, a partir de estudos sociométricos empreendidos por Jacob L. Moreno, que culminaram no desenvolvimento de métodos inovadores para análise das relações dentro de pequenos grupos, ganhando corpo conceitual nas décadas de 1940 e 1950. Entre as décadas de 1960 e 1980, a abordagem agregou significativa densidade teórica e metodológica (PROCOPIUCK; FREY, 2007). Nesse período, a análise de redes sociais desenvolveu uma gama considerável de conceitos e técnicas que dão suporte à captação e representação das relações entre atores sociais com vistas a representálas e interpretá-las estruturalmente sob a ótica das redes (WELLMAN, 1988).

A palavra sociometria significa medição do social, sendo o conjunto de técnicas para investigar, medir, estudar relações, contatos e processos vinculares que se manifestam nos diversos grupos sociais (CAMOSSA; LIMA, 2011). Este termo, estrategicamente, tem por finalidade abordar os fenômenos sociais, buscando entender as implicações dos padrões de relacionamento em uma rede com intuito de ampliar o seu desempenho e o seu desenvolvimento (GUARNIERI, 2010).

A análise de redes sociais tem como foco os vínculos relacionais (do inglês relational tie), ou seja, considera atores ligados uns aos outros por vínculos sociais. Podemos identificar os seguintes tipos de vínculos: social (amizade); por associação e afiliação (clubes e associações); por interação profissional (trabalho, científica, tecnológica etc.); física (rede Internet, cidade, bairro etc.); biológico (família); dentre outros.

Todas as organizações precisam ser analisadas como uma entidade social, pois estão inseridas em redes de importantes aspectos sociais (formais ou informais), nas quais as ações (atitudes e comportamento) podem ser explicadas em termos da posição na rede dos atores. Desta forma, usar técnicas de análise para avaliar o padrão dos relacionamentos mostra-se eficiente para organizações medirem as variáveis que refletem a estrutura global das relações com outros agentes (NOHRIA, 1992).

Dentro desta abordagem, uma importante variável que vem ganhando espaço em estudos de redes sociais refere-se à centralidade dos atores, definindo o poder e a influência que determinados atores podem assumir no relacionamento com outros atores, assim as redes são continuamente moldadas pelas ações de atores, que, por sua vez, são condicionados pela posição estrutural em que se encontram (BORGATTI; FOSTER, 2003; NOHRIA, 1992).

Entre os principais indicadores estruturais de redes, destacam-se: a) densidade - razão entre as relações existentes e as relações possíveis; b) grau de centralidade - mede o quanto um ator está centralizado em relação aos demais atores da rede. Dependendo do fluxo, pode ser considerado como grau de entrada (quando alguém cita a instituição) ou saída (quando a instituição cita outra); c) grau de proximidade - mede o 
quanto um ator está próximo ou pode alcançar os demais atores da rede. $\mathrm{O}$ ator com o maior grau de proximidade é aquele que atravessa o menor número de laços para chegar a todos os outros na rede; e d) grau de intermediação mede o quanto um ator exerce papel de mediador sob outros atores, ou seja, situa-se em uma posição na qual os demais atores da rede comumente devem passar para alcançar o outro (LIMIEUX; QUIMET, 2008).

Ademais, segundo Fletcher Júnior (2011), a análise de redes sociais permite uma solução flexível e poderosa, não só para compreender os fatores que influenciam as conexões, mas, também, para fornecer estimativas mais confiáveis e persistentes da sociedade em face dos limites dos dados.

\section{Consórcio das universidades do sul-sudeste de Minas Gerais}

A iniciativa pioneira da criação do Consórcio das Universidades do Sul-Sudeste de Minas Gerais, composto pelas seguintes instituições: Universidade Federal de Alfenas (UNIFAL-MG), Universidade Federal de Itajubá (UNIFEI), Universidade Federal de Juiz de Fora (UFJF), Universidade Federal de Lavras (UFLA), Universidade Federal de Ouro Preto (UFOP), Universidade Federal de São João Del-Rei (UFSJ) e Universidade Federal de Viçosa (UFV), surgiu em 2010.

Inicialmente, o consórcio reuniria mais de 250 cursos de graduação, oferecendo mais de 13 mil vagas e atendendo a mais de 43 mil alunos matriculados na graduação. Já na pós-graduação, ofereceria 121 programas e 175 cursos, sendo dois com conceito 7 (máximo), cinco com conceito 6 e 15 com conceito 5 (todos estes considerados de excelência), contando com mais de 5 mil alunos matriculados. Juntas, as Universidades do Sul-Sudeste de Minas Gerais apresentam mais de 50 citações na rede ISI Web of Knowledge. Com relação ao pessoal, o consórcio reúne 3.784 docentes e 5.620 técnicos administrativos. De acordo com a CAPES, o número de docentes permanentes atuando na pós-graduação chega a 1.553 (UNIVERSIDADE FEDERAL DE LAVRAS, 2010).

Neste contexto, o consórcio representa uma mudança de postura, passando do modelo de competição para um ambiente de cooperação entre as universidades consorciadas, preservando a autonomia de cada instituição, possibilitando que as bibliotecas possam, também, acompanhar novas tendências e desenvolver várias atividades de forma compartilhada (UNIVERSIDADE FEDERAL DE LAVRAS, 2010).

\section{Aspectos metodológicos}

Esta pesquisa parte de um estudo de caso que, na visão de Yin (2001), representa uma investigação empírica e compreende um método abrangente, com a lógica do planejamento, da coleta e da análise de dados. Segundo esse autor, o estudo de caso único pode ser classificado 
como crítico, revelador ou em profundidade e, assim, foi utilizado, nesta pesquisa, o estudo de caso do Consócio das Universidades Federais do Sul-Sudeste de Minas Gerais, cujo objetivo é analisar a estrutura de rede formada, assim como os atores centrais identificados.

O primeiro passo da pesquisa deu-se através da identificação dos responsáveis pelas bibliotecas das universidades participantes do consórcio. Em seguida, foi aplicado um questionário semiestruturado, através do Google docs, composto de duas partes: a primeira de identificação da instituição e a segunda de questões técnicas objetivas, que proporcionavam indicar quais os laços de relacionamentos entre as bibliotecas.

O método de análise de redes consiste em um conjunto finito de atores e as relações estabelecidas dos mesmos (WASSERMAN; FAUST, 1994). Este método permite a análise e comparação de distintas relações e fluxos entre os atores da rede por meio das seguintes variáveis de estudos: 1) troca de informações, documentos, planos e outros; 2) conversas informais sobre a dinâmica das instituições; 3) procura por profissionais antes de tomar uma decisão chave; 4) procura por profissionais por sua experiência técnica; 5) acompanhamento e implantação de inovação; e 6) busca dos sites das bibliotecas participantes para padronização de procedimentos técnicos/site.

Os dados recolhidos, no mês de julho de 2012, foram organizados e representados através de grafos. Para o cálculo das medidas de análise, utilizou-se dois softwares: Ucinet $\AA$ (BORGATTI; EVERETT; FREEMAN, 2002), para entrada e manipulação dos dados, e o NetDraw $\circledR$ (BORGATTI, 2002), que, acompanhado do primeiro, permitiu a visualização do mapa da rede, assim como a identificação da densidade do grau de centralidade da rede.

Os atores da rede foram representados pela sigla de cada instituição. Seguindo recomendações de Alejandro e Norman (2006), os laços foram representados como (0) ausente ou (1) presente, ou seja, calculados simplesmente a partir de uma relação binomial (existência ou ausência de vínculos) através de uma matriz quadrática.

Por fim, com o objetivo de medir os laços fortes e fracos das instituições analisadas, assim como os buracos estruturais existentes na rede geral formada pelas variáveis em estudo, foi construída uma matriz ponderada, evidenciando as relações acumuladas em todas as redes das variáveis pesquisadas.

Nessa matriz, a definição da presença ou ausência de relações foi ponderada, conforme o número de relacionamentos evidenciados nas redes anteriores, no qual, quanto maior o número de relações, mais forte - laço na rede. Como critério de classificação sobre a força dos laços, considera-se neste artigo que relações com escore 6 ou 5, em pelo menos uma das direções, assume um tipo de "laço muito forte"; escore 4 ou 3, em pelo menos uma das direções, assume um tipo de "laço forte"; e escore 2 ou 1 em pelo menos uma das direções assume um tipo de "laço fraco". 
Ademais, cabe ressaltar que a análise sociométrica utilizada neste estudo foi adotada como uma ferramenta analítica dos padrões de interações entre as bibliotecas do Consórcio das Universidades Federais do Sul-Sudeste de Minas Gerais, com foco nas relações sociais entre os atores e não em seus atributos.

\section{Análise dos dados}

Para estruturar e analisar a rede, os bibliotecário-documentalistas diretores ou responsáveis pelas bibliotecas foram questionados em seis itens, sendo estes analisados a partir do grau de centralidade, o que, segundo Alejandro e Norman (2006), representa o número de conexões existentes em relação ao total possível, ou seja, o quão bem conectado cada indivíduo está na rede.

O primeiro item pesquisado identificou quais bibliotecas do consórcio trocam informações, documentos, planos e/ou outros recursos entre si. Desta forma, foi detectado como ator central dessa rede a UFJF, com grau de entrada e saída normalizada de $83,3 \%$, seguido por UFLA e UFOP, com grau de entrada e saída normalizada de 50\%. Em situação oposta, em condições mais periféricas, encontram UNIFEI, UFSJ e UNIFAL, com grau de entrada e saída normalizada de 16,7\%, conforme apresentado pela Figura e Tabela 1.

Figura 1 - Rede 1 - Troca de informações, documentos, planos e outros

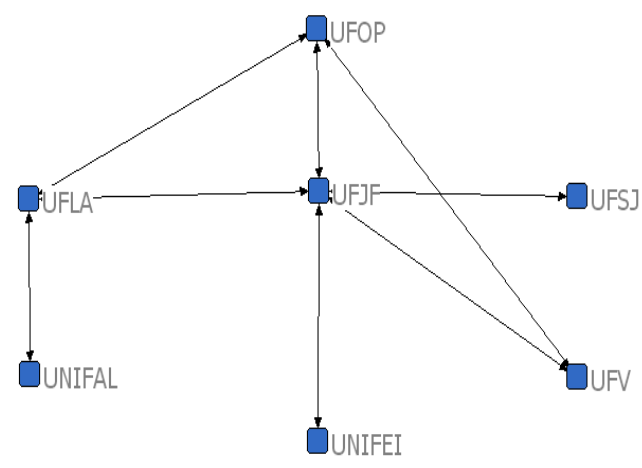

Fonte: Dados da pesquisa.

Tabela 1 - Grau de centralidade de Freeman's

\begin{tabular}{|c|c|c|c|c|}
\hline & 1 & 2 & 1 & 2 \\
\hline & Grau de saída & $\begin{array}{l}\text { Grau de } \\
\text { entrada }\end{array}$ & $\%$ & $\%$ \\
\hline UFJF & 5.0 & 5.0 & 83.3 & 83.3 \\
\hline UFLA & 3.0 & 3.0 & 50.0 & 50.0 \\
\hline UFOP & 3.0 & 3.0 & 50.0 & 50.0 \\
\hline UFV & 2.0 & 2.0 & 33.3 & 33.3 \\
\hline UNIFEI & 1.0 & 1.0 & 16.7 & 16.7 \\
\hline UFSJ & 1.0 & 1.0 & 16.7 & 16.7 \\
\hline UNIFAL & 1.0 & 1.0 & 16.7 & 16.7 \\
\hline
\end{tabular}

Fonte: Dados da pesquisa.

O segundo item questionado foi sobre quais profissionais das bibliotecas do consórcio os dirigentes mantinham conversas informais 
sobre a dinâmica da sua instituição. A UFJF, com grau de saída normalizada de $83,3 \%$ e de entrada de $50 \%$, foi identificada, novamente, como o ator mais central nessa variável, seguida por UFLA, com grau de saída normalizada de $50 \%$ e de entrada de 33,3\% e a UFOP, com grau de saída normalizada de $50 \%$ e de entrada de $66,7 \%$. Na outra extremidade das interações relacionadas a essa variável, a UNIFEI foi considerada como um ator não-conexo, sem interações de qualquer sentido (Figura e Tabela 2).

Figura 2 - Rede 2 - Conversas informais sobre a Dinâmica entre as instituições

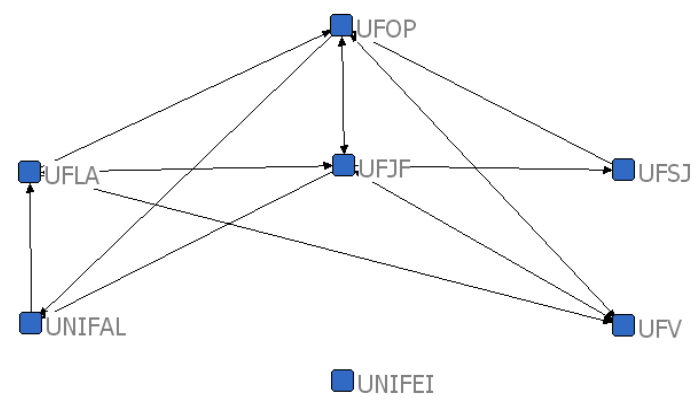

Fonte: Dados da pesquisa.

Tabela 2 - Grau de centralidade de Freeman's

\begin{tabular}{l|c|c|c|c}
\hline & 1 & 2 & 1 & 2 \\
\hline & Grau de saída & $\begin{array}{c}\text { Grau de } \\
\text { entrada }\end{array}$ & $\%$ & $\%$ \\
\hline UFJF & 5.0 & 3.0 & 83.3 & 50.0 \\
\hline UFLA & 3.0 & 2.0 & 50.0 & 33.3 \\
\hline UFOP & 3.0 & 4.0 & 50.0 & 66.7 \\
\hline UFV & 2.0 & 3.0 & 33.3 & 50.0 \\
\hline UFSJ & 1.0 & 1.0 & 16.7 & 16.7 \\
\hline UNIFAL & 1.0 & 2.0 & 16.7 & 33.3 \\
\hline UNIFEI & 0.0 & 0.0 & 0.0 & 0.00 \\
\hline
\end{tabular}

Fonte: Dados da pesquisa.

O terceiro item pesquisado refere-se à procura por profissionais de quais bibliotecas os dirigentes já haviam procurado antes de tomar uma decisão chave, sendo diagnosticado que a UFJF, com grau de entrada normalizada de $83,3 \%$, novamente foi a mais central, desempenhando um papel fundamental na estrutura dessa rede, pois representa maior interação na rede na busca por decisões chaves, seguida por UNIFAL e UFV. Como atores mais periféricos, identificamos a UNIFEI, UFOP e UFS], como as instituições que menos interagem na rede, conforme graus de entradas e saídas apresentados na figura e tabela 3. 
Figura 3 - Rede 3 - Profissional de qual biblioteca foi procurado antes de tomar uma decisão chave

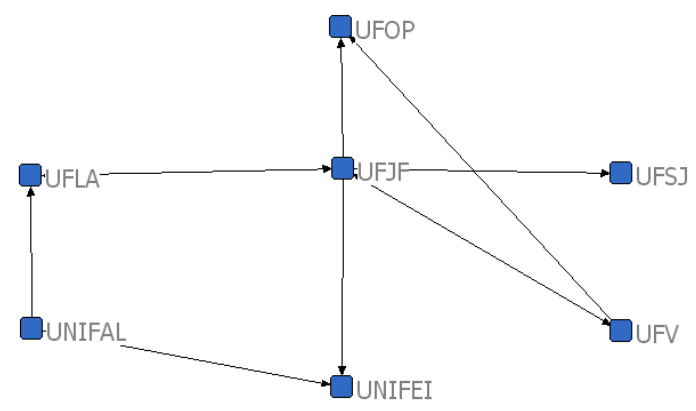

Fonte: Dados da pesquisa.

Tabela 3 - Grau de centralidade de Freeman's

\begin{tabular}{l|c|c|c|c}
\hline & 1 & 2 & 1 & 2 \\
\hline UFJF & $\begin{array}{c}\text { Grau de } \\
\text { saída }\end{array}$ & $\begin{array}{c}\text { Grau de } \\
\text { entrada }\end{array}$ & $\%$ & $\%$ \\
\hline UNIFAL & 2.0 & 2.0 & 83.3 & 33.3 \\
\hline UFV & 2.0 & 0.0 & 33.3 & 0.0 \\
\hline UFLA & 1.0 & 2.0 & 33.3 & 16.7 \\
\hline UNIFEI & 0.0 & 2.0 & 0.0 & 33.3 \\
\hline UFOP & 0.0 & 2.0 & 0.0 & 33.3 \\
\hline UFSJ & 0.0 & 1.0 & 0.0 & 16.7 \\
\hline
\end{tabular}

Fonte: Dados da pesquisa.

No quarto item pesquisado, os dirigentes das bibliotecas do consórcio foram questionados sobre o profissional de qual biblioteca já haviam procurado por sua experiência técnica. A UFJF, com grau de saída normalizada de $100 \%$ e entrada de 66,7\%, foi novamente considerada a biblioteca mais central, seguida pela UFLA e UFOP, com grau de saída normalizada de 33,3\% e saída de 16,7\%. No outro extremo da rede, localiza-se a UNIFAL, com nenhum grau de saída e grau de entrada normalizada de 33,3\%, conforme apresentado pela Figura e Tabela 4.

Figura 4 - Rede 4 - Profissional de qual biblioteca já havia procurado por sua experiência técnica

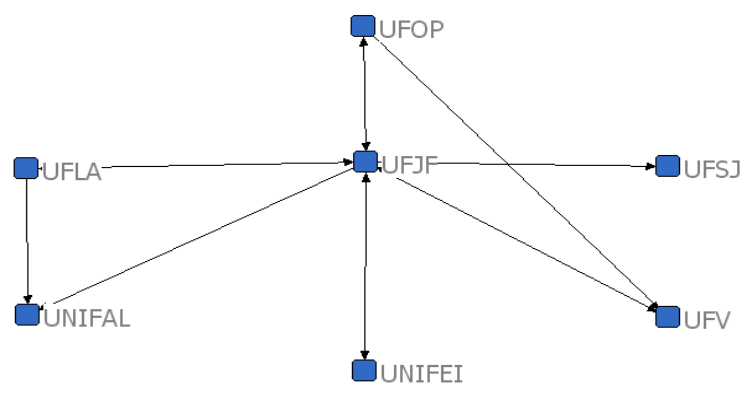

Fonte: Dados da pesquisa. 
Tabela 4 - Grau de centralidade de Freeman's

\begin{tabular}{l|c|c|c|c}
\hline & 1 & 2 & 1 & 2 \\
\hline & Grau de saída & $\begin{array}{c}\text { Grau de } \\
\text { entrada }\end{array}$ & $\%$ & $\%$ \\
\hline UFJF & 6.0 & 4.0 & 100.0 & 66.7 \\
\hline UFLA & 2.0 & 1.0 & 33.3 & 16.7 \\
\hline UFOP & 2.0 & 1.0 & 33.3 & 16.7 \\
\hline UFV & 1.0 & 2.0 & 16.7 & 33.3 \\
\hline UNIFEI & 1.0 & 1.0 & 16.7 & 16.7 \\
\hline UFSJ & 0.0 & 1.0 & 0.0 & 16.7 \\
\hline UNIFAL & 0.0 & 2.0 & 0.0 & 33.3 \\
\hline
\end{tabular}

Fonte: Dados da pesquisa.

No quinto item pesquisado, foi avaliado com qual biblioteca do consórcio os dirigentes trocam informações, quando se trata de implantação de inovação. Nessa variável, a UFV, com grau de saída normalizada de $33,3 \%$ e entrada de $16,7 \%$ e a UFJF, com grau de saída normalizada de $33,3 \%$ e entrada de $50 \%$, foram consideradas as mais centrais. No outro extremo, destaca-se a UNIFEI, que não foi mencionada em nenhum tipo de interação, conforme apresentado pela Figura e Tabela 5.

Figura 5 - Rede 5 - Referência para implantação de inovação

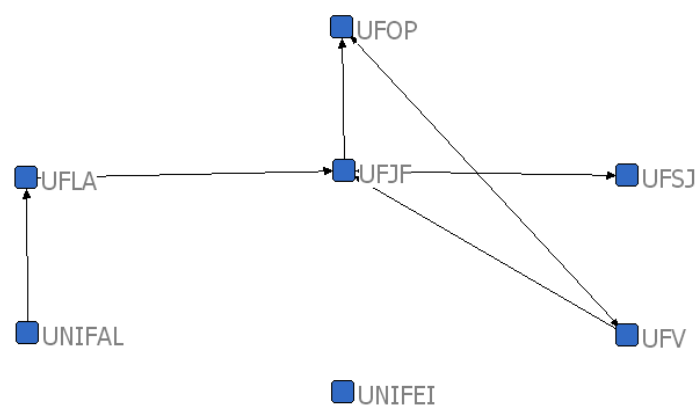

Fonte: Dados da pesquisa.

Tabela 5 - Grau de centralidade de Freeman's

\begin{tabular}{l|c|c|c|c}
\hline & 1 & 2 & 1 & 2 \\
\hline & Grau de saída & $\begin{array}{c}\text { Grau de } \\
\text { entrada }\end{array}$ & $\%$ & $\%$ \\
\hline UFV & 2.0 & 1.0 & 33.3 & 16.7 \\
\hline UFJF & 2.0 & 3.0 & 33.3 & 50.0 \\
\hline UFOP & 1.0 & 2.0 & 16.7 & 33.3 \\
\hline UNIFAL & 1.0 & 0.0 & 16.7 & 0.0 \\
\hline UFSJ & 1.0 & 1.0 & 16.7 & 16.7 \\
\hline UFLA & 1.0 & 1.0 & 16.7 & 16.7 \\
\hline UNIFEI & 0.0 & 0.0 & 0.0 & 0.0 \\
\hline
\end{tabular}

Fonte: Dados da pesquisas

Na última variável de estudo, foram questionados sobre o site de busca de qual biblioteca os dirigentes visitam para consulta, análise ou referências com relação aos procedimentos técnicos. Segundo a análise sociométrica, as páginas da UFV e UFJF, com grau de saída normalizada de $100 \%$ e entrada de $66,7 \%$, foram consideradas as mais centrais. $\mathrm{Na}$ outra extremidade, a UFLA foi considerada o ator mais periférico, com 
grau de saída normalizada de 16,7 e entrada de 50\%, conforme apresentado pela Figura e Tabela 6.

Figura 6 - Rede 6 - Sites de busca de quais bibliotecas os dirigentes visitam para procedimentos técnicos

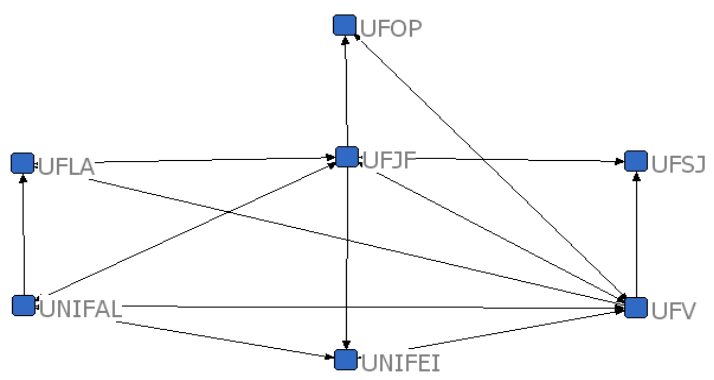

Fonte: Dados da pesquisa.

Tabela 6 - Grau de centralidade de Freeman's

\begin{tabular}{l|c|c|c|c} 
& 1 & 2 & 1 & 2 \\
\hline UFV & Grau de saída & $\begin{array}{c}\text { Grau de } \\
\text { entrada }\end{array}$ & $\%$ & $\%$ \\
\hline UFJF & 6.0 & 4.0 & 100.0 & 66.7 \\
\hline UNIFAL & 4.0 & 4.0 & 100.0 & 66.7 \\
\hline UNIFEI & 2.0 & 3.0 & 66.7 & 50.0 \\
\hline UFOP & 1.0 & 3.0 & 33.3 & 50.0 \\
\hline UFSJ & 1.0 & 2.0 & 16.7 & 33.3 \\
\hline UFLA & 1.0 & 2.0 & 16.7 & 33.3 \\
\hline
\end{tabular}

Fonte: Dados da pesquisa.

Por fim, com o propósito de analisar a estrutura geral da rede, foi gerada, através do software NetDraw ${ }^{\circledR}$, uma rede ponderada das bibliotecas que compõem o Consórcio das Universidades Federais do SulSudeste de Minas Gerais. Esta rede representa o conjunto das variáveis individuais analisadas anteriormente, representando o total de relações acumuladas em relação a: 1) troca de informações, documentos, planos e outros; 2) conversas informais sobre a dinâmica das instituições; 3) procura por profissionais antes de tomar uma decisão chave; 4) procura por profissionais por sua experiência técnica; 5) acompanhamento e implantação de inovação; e 6) busca dos sites das bibliotecas participantes para padronização de procedimentos técnicos/site. Sendo calculados através do software UCINET®, os indicadores de centralidade (entrada e saída) destas relações acumuladas, conforme apresentado a seguir. 
Figura 7 - Rede 7 - Relações ponderadas das bibliotecas do Consócio SulSudeste de Minas Gerais

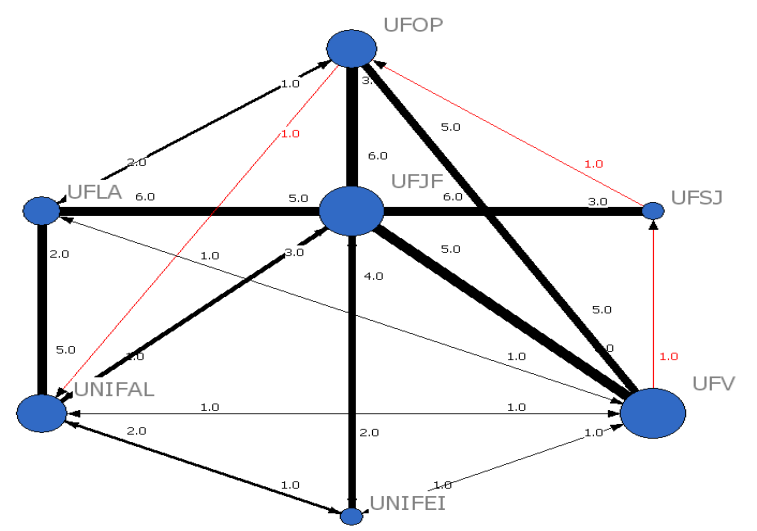

Fonte: Dados da pesquisa.

Tabela 7 - Grau de centralidade de Freeman's

\begin{tabular}{l|c|c|c} 
& $\begin{array}{c}\text { Grau de } \\
\text { saída }\end{array}$ & $\begin{array}{c}\text { Grau de } \\
\text { entrada }\end{array}$ & $\begin{array}{c}\text { Total de } \\
\text { relações }\end{array}$ \\
\hline UFJF & 29.0 & 21.0 & 50.0 \\
\hline UFV & 15.0 & 13.0 & 28.0 \\
\hline UFLA & 11.0 & 12.0 & 23.0 \\
\hline UFOP & 10.0 & 14.0 & 24.0 \\
\hline UNIFAL & 9.0 & 8.0 & 17.0 \\
\hline UNIFEl & 4.0 & 7.0 & 11.0 \\
\hline UFSJ & 4.0 & 7.0 & 11.0 \\
\hline
\end{tabular}

Fonte: Dados da pesquisa.

Neste sentido, percebe-se "laços muito fortes" (representados pelas linhas com maior espessura) da UFJF com as instituições: UFLA, UFOP, UFSJ e UFV; da UFLA com as instituições: UFJF e UNIFAL; da UFOP com as instituições: UFJF e UFV; da UFV com as instituições UFJF e UFOP; e, por fim, da UFSJ com a UFJF. Percebe-se, ainda, "laços fortes" (representados pelas linhas com espessura média) entre a UFJF com a UNIFEI e UNIFAL. Já em relação aos laços fracos (representados pelas linhas com menor espessura), percebem-se relações da UNIFAL com UFOP; UFV com UNIFEI; da UFSJ com UFOP e UFV; da UFOP com UNIFAL e UFSJ; da UFV com UFSJ, UNIFEI, UNIFAL e UFLA; da UNIFEI com UFV e UNIFAL; e, por fim, da UFLA com UFOP e UFV.

Corroborando as informações apresentadas nas redes anteriores, nota-se que a UFJF apresenta uma posição central na rede, pois identificou-se quatro laços muito fortes e dois laços fortes, além de não apresentar laços fracos com nenhuma das instituições analisadas, representando um total de 50 relações ( 29 de entrada e 21 de saída), assumindo uma posição dominante na rede, pois possui conexões com todos os demais participantes, o que demonstra ter maior poder e influência na rede (LEMIEUX; OUIMET, 2008; ALEJANDRO; NORMAN, 2006; BORGATTI; FOSTER, 2003; NOHRIA, 1992). 
Por outro lado, percebe-se que a UNIFEI encontra-se em uma posição mais periférica na rede, pois não apresenta "laços muito fortes" com nenhuma instituição, apenas um "laço forte" (com a UFJF) e dois "laços fracos" (com UNIFAL e UFV), representando um total de 11 relações (4 de entrada e 7 de saída), além de se relacionar com UFSJ, UFOP e UFLA.

Assim, a centralidade de cada ator na rede pode ser observada no tamanho dos nós representados na Figura 7, com destaque para UFJF e UFV conectadas com todas as instituições; UNIFAL e UFOP conectadas a outras 5 instituições; UFLA conectada a outras 4 instituições e, por fim, UNIFEI e UFS] conectadas com outras três instituições. Percebe-se, ainda, pela rede ponderada a existência de relações orientadas (em vermelho), quando a relação se apresenta em um único sentido, e não orientadas (em preto), com relações estabelecidas em sentidos duplos (LEMIEUX; OUIMET, 2008), em que a incidência de laços mais fortes é mais evidenciada em relações não orientadas, estando as relações orientadas formadas por laços mais fracos, tendo como consequência menor troca de informações entre atores.

Por fim, nota-se que a rede analisada apresenta "buracos estruturais", o que, segundo Burt (1992), representa a existência de diferentes agentes desconectados em uma estrutura social. Neste sentido, evidenciam-se buracos estruturais entre: 1) UFOP e UNIFEI; 2) UFSJ e UFLA, UNIFAL e UNIFEI; 3) UNIFAL com UFSJ; 4) UNIFEI e UFLA, UFOP e UFS]; 5) e UFLA com UNIFEI e UFSJ. Diversos autores indicam que um buraco estrutural representa a oportunidade de agenciar o fluxo de informação e acessar pessoas relevantes para aproveitamento de oportunidades de negócios, quando assumidos por atores centrais. Neste caso, é comum uma condição de "tertius Gaudens", ou seja, atores fora dos buracos estruturais podem assumir uma posição de coordenação da rede pelo maior volume de informações e maior diversidade de acesso, gerando novas oportunidades de controle (BURT, 1992; LEMIEUX; OUIMET, 2008). Neste sentido, percebe-se que a UFJF e UFV assumem maior coordenação da rede, pois se encontram em uma posição favorável aos buracos estruturais existentes, visto que podem se relacionar e intermediar relações com as demais instituições da rede. 


\section{Considerações finais}

Partindo de referenciais teóricos, que consideram as redes interorganizacionais como um importante fator de competitividade pelos benefícios gerados pela troca de experiências, acesso a novas tecnologias, maior acesso a informações e abertura a novos processos inovativos (BENGTSON; KOCK, 1999; BRITTO, 2002; MA, 2004; LACOSTE, 2012; CAPALDO, 2007), foi possível identificar, por meio da análise sociométrica na rede de bibliotecas do Consórcio das Universidades Federais do sulsudeste de Minas Gerais, algumas disparidades de interação entre as instituições analisadas.

De um lado, ficou evidente a centralidade da UFJF em todas as variáveis pesquisadas, representando que a mesma encontra-se em uma posição favorável para trocas de informações, busca por informações técnicas e inovação na rede, possuindo uma proximidade relacional com todas as instituições participantes, o que a coloca em uma posição de referência quanto à coordenação da rede. Já no outro extremo, evidenciase que a UFSJ e a UNIFEI encontram-se em posições mais periféricas, não interagindo de forma intensiva com as demais instituições da rede, quanto às variáveis pesquisadas.

Neste sentido, à luz das teorias dos laços fortes e fracos de Granovetter (1973), percebe-se pela rede ponderada uma centralidade maior da UFJF e UFV, o que, ao menos na perspectiva teórica, pode favorecer um maior grau de flexibilidade e inovação. Por outro lado, relações mais esparsas de outras instituições têm apresentado buracos estruturais na rede, o que, segundo Burt (1992) e Hargadon e Sutton (1997), podem provocar a perda de benefícios e novas oportunidades.

Ressalta-se que o estudo em questão apresenta alguns vieses, na medida em que as relações analisadas se restringem apenas a rede formada por instituições vinculadas ao consórcio de bibliotecas do sulsudeste de Minas Gerais, não considerando a interação destas com dirigentes de outras bibliotecas não participantes da rede. Outra limitação encontrada refere-se à coleta de dados que, por coincidir com o período de greve (2012), gerou barreiras para obter respostas de questões que envolviam setores mais técnicos e específicos. Diante destas limitações, surge a necessidade de novos estudos empíricos através de uma estrutura de rede mais ampla e complementada com outros indicadores estruturais não utilizados neste artigo, como os indicadores de proximidade, intermediação e densidade da rede, permitindo, assim, análises mais adequadas de investigação e estratégias de cooperação mais efetivas.

Ademais, 0 estudo mostra-se relevante aos dirigentes das instituições participantes do consórcio das bibliotecas do sul-sudeste de Minas Gerais, pois permite analisar a estrutura da rede formada, assim como os atores centrais nas diversas variáveis abordadas ao longo deste artigo, sendo possível identificar em que posições se encontram na rede, os buracos estruturais existentes, quais instituições possuem laços fortes, 
fracos, ou ainda ausência de relações, permitindo desta forma elaborar estratégias para usufruir melhor da estrutura da rede, buscando novos benefícios e informações capazes de gerar inovações e maior competitividade através de ações coletivas promovidas por relações interorganizacionais.

\section{Referências}

AARSTAD, J. Do structural holes and network connectivity really affect entrepreneurial performance? Journal of Entrepreneurship, New Delhi, v. 21, n. 2, p. 253-268, Sept. 2012.

ALEJANDRO, V. A. O.; NORMAN, A. G. Manual introdutório à análise de redes sociais. Toluca: Universidad Autónoma del Estado de México, 2006.

BALESTRIN, A.; VERSCHOORE, J. R.; REYES JÚNIOR, E. O campo de estudo sobre rede de cooperação interorganizacional no Brasil. Revista de Administração Contemporânea, Curitiba, v. 14, n. 3, pp. 458-477, mai./jun, 2010.

BALESTRO, M. V. Confiança em rede: a experiência da rede de estofadores do pólo moveleiro de Bento Gonçalves. 2002. $118 \mathrm{f}$. Dissertação (Mestrado em Administração) - Universidade Federal do Rio Grande do Sul, Porto Alegre, 2002.

BENGTSON, M.; KOCK, S. Cooperation and competition in relationships between competitors in business networks. Journal of business \& industrial Marketing, Santa Barbara, v. 14, n. 3, p. 178-193, 1999.

BORGATTI, S. P. Netdraw network visualization. Harvard: Analytic Technologies, 2002.

BORGATTI, S. P.; EVERETT, M. G.; FREEMAN, L. C. Ucinet for Windows: software for social network analysis. Harvard: Analytic Technologies, 2002.

BORGATTI, S. P.; FOSTER, P. C. The network Paradigm in organizational research: a review and typology. Journal of Management, Stillwater, $v$. 29, n. 6, p. 991-1013, 2003.

BRITTO, J. Cooperação interindustrial e redes de empresas. In: KUPFER, D.; HASENCLEVER, L. (Org.). Economia industrial: fundamentos teóricos e práticas no Brasil. Rio de Janeiro: Campus, 2002.

BURT, R. S. The social structure of competition. In: NOHRIA, N.; ECCLES, R. G. Networks and organizations: structure, form and action. Boston: Boston Harvard Business School, 1992. cap. 2, p. 57-91.

BURT, R.; KILDUFF, M.; TASSELLI, S. Social network analysis: foundations and frontiers on advantage. Annual Review of Psychology, Palo Alto, v. 64, p. 527-547, 2013. 
CAMOSSA, D. A.; LIMA, N. S. T. O psicodrama e sua contribuição para a saúde mental. Revista de Ciências da Educação, Americana, v. 25, n. 8, p. 1-12, jul./dez. 2011.

CAPALDO, A. Network structure and innovation: the leverage of a dual network as a distinctive relational capability. Strategic Management Journal, Hoboken, v. 28, p. 585-608, 2007.

CASANUEVA, C.; CASTRO, I.; GALÁN, J. L. Informational networks and innovation in mature industrial clusters. Journal of Business Research, Athens, v. 66, n. 5, p. 603-613, May 2013.

FAGERBERG, J.; MOWERY, D. C.; NELSON, R. R. Network of innovation. In: FAGERBERG, J.; MOWERY, D. C.; NELSON, R. R. The oxford handbook of innovation. Oxford: Oxford University, 2006. cap. 3, p. 56-85.

FOMBRUN, C. Strategies for network research in organisatons. Academy of Management Review, Mississippi, v. 7, n. 2, p. 280-291, 1982.

FLETCHER JUNIOR, R. J. et al. Social network models predict movement and connectivity in ecological landscapes. Proceedings of the National Academy of Sciences of the Estados Unidos da América, Washington, v. 108, n. 48, p. 19282-19287, 2011.

GRANDORI, A.; SODA, G. Inter-firm network: antecedents, mechanisms and forms. Organization Studies. Organizations Studies, Berlin, v. 16, n. 2, p. 183-214, 1995.

GRANOVETTER, M. S. The strength of weak ties. American Journal of Sociology, Chicago, v. 6, p 1360-1380, 1973.

GUARNIERI, P. Análise de redes sociais. 2010. Slides. Disponível em: <http://pt.slideshare.net/fhguarnieri/anlise-de-redes-sociais-teoria-eprtica $>$. Acesso em: 15 dez. 2013.

HARGADON, A.; SUTTON, R. I. Technology brokering and innovation in a product development firm. Administrative Science Quarterly, Ithaca, v. 42, p. 716-49, 1997.

LACOSTE, S. "Vertical coopetition": the key account perspective. Industrial Marketing Management, New York, v. 41, p. 649-658, 2012.

LEMIEUX, V.; OUIMET, M. Análise estrutural das redes sociais. Lisboa: Instituto Piaget, 2008. 116 p.

MA, H. Toward global competitive advantage: creation, competition, cooperation, and co-option. Management Decision, York, GB, v. 42, n. 7, p. 907-924, 2004.

NOHRIA, N. Is a network perspective a useful way of studying organizations? In: NOHRIA, N.; ECCLES, R. G. Networks and organizations: structure, form, and action. Boston: Harvard Business School, 1992. p. 1-22. 
PROCOPIUCK, M.; FREY, K. Redes sociotécnicas de difusão social de TICs em Porto Alegre e Curitiba. In: ENCONTRO DA ANPAD, 31., 2007, Rio de Janeiro. Anais... Rio de Janeiro: Anpad, 2007. 1 CD-ROM.

UNIVERSIDADE FEDERAL DE LAVRAS. Consórcio de universidades é discutido na Assembléia. Lavras, 2010. Disponível em: <http://www.ufla.br/ascom/index.php/2010/12/ consorcio-deuniversidades-e-discutido-na-assembleia/>. Acesso em: 8 mar. 2013.

WASSERMAN, S.; FAUST, K. Social network analysis: methods and applications. Cambridge: Cambridge University, 1994.

WELLMAN, B. Structural analysis: from method and metaphor to theory and substance. In: WELLMAN, B.; STEVE, B. (Orgs.). Social structures: a network approach. Cambridge: University, 1988. cap. 2, p. 19-61.

WU, M. From weak ties to strong ties: community vs. social networks 3. Lithosphere, 22 jun. 2012. Disponível em: <http://lithosphere.lithium.com/t5/science-of-social-blog/From-WeakTies-to-Strong-Ties-Community-vs-Social-Networks-3/ba-p/6834>. Acesso em: 12 mar. 2013.

YIN, R. Estudo de caso: planejamento e métodos. 2. ed. Porto Alegre: Bookman; 2001. 